\title{
Changes in antennal gene expression underlying sensory system maturation in Rhodnius prolixus
}

\section{Jose Manuel Latorre-Estivalis ${ }^{1 *}$; Ewald Große-Wilde ${ }^{2,3^{*}}$; Gabriel da Rocha Fernandes $^{4}$, Bill S. Hansson ${ }^{2}$ and Marcelo Gustavo Lorenzo ${ }^{5 \S}$.}

(1) Instituto de Fisiología, Biología Molecular y Neurociencias, Universidad de Buenos Aires - CONICET, Buenos Aires, Argentina

(2) Department of Evolutionary Neuroethology, Max Planck Institute for Chemical Ecology, Jena, Germany. (3) Present address: Faculty of Forestry \& Wood Sci, Excellent Team for Mitigation, Czech University Life Sci, Prague, Czech Republic

(4) Plataforma de Bioinformática, Instituto René Rachou - FIOCRUZ, Belo Horizonte, Minas Gerais, Brazil.

(5) Vector Behavior and Pathogen Interaction Group, Instituto René Rachou - FIOCRUZ, Belo Horizonte, Minas Gerais, Brazil.

* These authors contributed equally to this work.

${ }^{\S}$ Corresponding author: marcelo.lorenzo@fiocruz.br

\section{Abstract \\ Background}

Triatomine bugs are the blood feeding insect vectors transmitting Chagas disease to humans, a neglected tropical disease that affects over 8 million people, mainly in Latin America. The behavioral responses to host cues and bug signals in Rhodnius prolixus are state dependent, i.e., they vary as a function of post-ecdysis age. At the molecular level, these changes in behavior are probably due to a modulation of peripheral and central processes. In the present study, we report a significant modulation of the 
expression of a large set of sensory-related genes. Results were generated by means of antennal transcriptomes of 5th instar larvae along the first week (days $0,2,4,6$ and 8) after ecdysis sequenced using the Illumina platform.

\section{Results}

Age induced significant changes in transcript abundance were established in more than 6,120 genes $(54,7 \%$ of 11,186 genes expressed) in the $R$. prolixus antenna. This was especially true between the first two days after ecdysis when more than 2,500 genes had their expression significantly altered. In contrast, expression profiles were almost identical between day 6 and 8, with only a few genes showing significant modulation of their expression. A total of 86 sensory receptors, odorant carriers and odorant degrading enzymes were significantly modulated across age points and clustered into three distinct expression profiles.

\section{Conclusions}

The set of sensory genes whose expression increased with age (profile 3) may include candidates underlying the increased responsiveness to host cues shown by $R$. prolixus during the first days after molting. For the first time, we describe the maturation process undergone at the molecular level by the peripheral sensory system is described in an hemimetabolous insect.

Key words: Age, transcriptome, antennae, Rhodnius prolixus, sensory genes

\section{Background}

Sensory performance is a fundamental component underlying the behavioral repertoire of an organism. It defines how precisely an animal can keep track of relevant events happening in its environment. The sensory sounding of the umwelt of insects is predominantly multimodal, i.e., the world is accessed through diverse 
senses and integration of sensory inputs frequently drives behavior [1, 2]. This seems particularly true for model kissing-bugs like Triatoma infestans and Rhodnius prolixus, which vector Trypanosoma cruzi, causative agent of Chagas disease, to humans [3]. Sensory neurons (SNs) covering diverse modalities concentrate mainly in the antennae of insects. These include olfactory, gustatory, thermo-, hygro-, or mechanosensory units that are finely tuned to detect their cognate stimuli. Nevertheless, the acuity and sensitivity of these sensory systems is not a static feature. As an example, it is well known that antennal responsiveness to odors presents a circadian variation in insects [4-7].

Insect behavioral responsiveness to communication signals and host cues can depend on factors such as age, nutritional or reproductive status. The responsiveness of sensory components informing decision-making neuropiles in the central nervous system is also affected by these factors. As an example, the imaginal moult involves a dramatic transformation of the insect that normally requires a few hours/days of final adjustments after ecdysis. This process is usually considered as a maturation process affecting behavior. Indeed, recently emerged mosquito females do not engage in host seeking, a behavior only expressed between the second and third day after their imaginal moult $[8,9]$. Insects that engorge on blood also commonly show dramatic changes in their behavior due to the physiological processes abruptly triggered by the blood meal $[10,11]$. After biting a host and becoming fully-engorged, blood-sucking insects quit host-seeking almost immediately [12]. In the case of mosquito females, two days later they initiate the search of a breeding site where to lay the egg batch [13]. Considering that such dramatic changes affecting foraging activities can be established, it seems feasible that they have molecular correlates both at the sensory periphery and in central processing centers.

While the detection of cues related to meal sources or oviposition sites is transiently needed for nurture or egg-laying, the detection of many other clues and signals needs to be permanently effective. This is the case for the recognition of predator-emitted 
odors, aversive compounds present in non-edible plants, alarm and aggregation pheromones. Some triatomines use aggregation signals present in their feces to mark their hiding places $[14,15]$. Aggregation responses to these signals are seen in all instars independently of age [16] and under most nutritional conditions [17]. Another example are the alarm signals emitted by adult triatomines when exposed to mechanical disturbance [18], as all instars need to present avoidance reactions to these pheromones in order to stay away from danger independently of their physiological condition. Therefore, one would expect that the molecular machinery committed to detect this type of stimuli should not vary significantly with age, neither should it change with nutritional status.

The developmental stage, age, and nutritional, and reproductive status of insects can affect SN sensitivity (reviewed by [19]). This is quite clear for kissing-bugs, insects that feed very sporadically on the blood of vertebrates that they steal in the still of the night. As an example, the antennae of $R$. prolixus are known to respond to ammonia with increasing electroantennogram amplitudes depending on starvation, but this is only true during the scotophase [20]. Adult mosquitoes also present significant changes in SN responsiveness as a function of post-emergence age [8, 21-23]. Peaks of responsiveness by SNs may also depend on reproductive status as observed in moths [24, 25]. Finally, it was recently shown that Dengue I infection can induce augmented olfactory responses in the antennae of infective mosquitoes [26], suggesting that pathogens can also modulate OSNs responsiveness.

Reports on gene expression profiles as a function of age, nutritional or mating status have proven that insect antennae go through a complex set of processes regulating the transcript abundance of diverse genes. Mosquito age after adult emergence affects the transcriptomic profile seen in the antennae of Ae. aegypti, with many sensory receptor-coding genes showing changes in their expression over the first five days of adult life in both sexes [8]. Furthermore, similar regulation of gene expression profiles in the antennae of females of the species is affected through the temporal 
interval of the first gonotrophic cycle and after ingestion of a blood meal [27]. Females of the malaria vector Anopheles gambiae also show clearly regulated expression changes in their antennae as a consequence of engorging on blood [28]. In the antennae of the hemimetabolous vector bug $R$. prolixus, the imaginal moult triggers a clear increase in the expression of many chemosensory genes compared to the last larval instar [29] suggesting that the antennae of adults become more sensitive to diverse odor molecules.

As Bodin et al. [30] have shown, triatomine responsiveness to host cues depends on age after ecdysis. While recently molted bugs are not attracted towards hostmimicking heat sources or $\mathrm{CO}_{2}$-added air currents, they show consistent responses to these host-related cues a week later. To date, it is not clear whether this is an exclusive consequence of a maturation process affecting peripheral and/or central components controlling responsiveness to host cues. We hypothesize that the abundance of olfactory, gustatory, thermo- and hygroreceptors potentially mediating the detection of host-related cues may vary through the maturation interval. In addition, we suggest that receptor proteins dedicated to the detection of aggregation and alarm pheromones should present a stable level of expression through the same interval. Our work aims to elucidate the extent to which sensory gene expression profiles change in $5^{\text {th }}$ instar bug antennae as a proxy to understand kissing-bug sensory plasticity at the peripheral level through the post-ecdysis maturation of bug behavior.

\section{Results}

\section{Exploratory analysis}

Samples from the five age points presented different expression profiles according to the PERMANOVA analysis plot (Fig. 1), indicating a strong effect of age on the sample distribution (PERMANOVA, $\mathrm{R}^{2}=0.42$, corrected $\mathrm{p}$-value $<0.01$, Additional file 1 : Table S1). In Fig. 1, replicates from days 0 and 2 appeared completely separated and paired 
comparisons with the other time points were all significant (Additional file 1: Table S1). On the other hand, replicates from days 4, 6 and 8 grouped together, with only days 4 and 8 differing significantly (Fig. 1 and Additional file 1: Table S1).

\section{Overall expression changes}

According to the GLM analysis performed in EdgeR, a total of 6,120 transcripts changed their antennal expression significantly during the first week of $R$. prolixus larval life. This represents $54,7 \%$ of 11,186 transcripts (after the HTSFilter processing step). The differentially expressed genes (DEGs) were identified based on False Discovery Rate (FDR) $<0.05$ with no fold-change threshold (Additional file 2: Table S2).

\section{Highly expressed sensory genes along age}

A consensus of 43 target genes was obtained from the top 50 most expressed sensory genes in each time point (Additional file 3: Table S3). As expected, odorant binding proteins (OBPs) and chemosensory proteins (CSPs) were the most abundant within this set, with 18 and 14 members, respectively. Regarding the other protein families, few members were highly expressed: RproGr27, Rprolr93, RproSnmp1a, RproPain, RproAmmT1, 4 odorant degrading enzymes (ODEs) and the PPK Rprrc013510.

\section{Pairwise comparisons}

Expression data from the different time points was compared using a pairwise analysis to identify ages at which the transcriptional changes occurred (Table 1). A total of 3,290 genes changed their expression significantly (s-values $<0.05$ and on an absolute fold-change threshold $>1.5$ ) in at least one of the comparisons performed. As we observed in the MA and PCoA plots, the greatest change in antennal expression occurred right after day 0 (with a total of 2,534 genes showing significant changes compared to a pool of the remaining days). The second greatest gene expression change was detected between day 0 vs. day 2 (1,738 genes representing $16,27 \%)$. These transcriptional changes are reflected in the corresponding MA-plots (Additional files 4 and 5: Figs. S1 and S2). The numbers of DEGs in the antennae decreased 
between subsequent ages, as well as the amplitude of their changes (Table 1 ). This reduction was more pronounced between days 6 and 8 , when only 80 genes changed their expression within a smaller range, supporting the clustering pattern observed in the MA and PCoA plots (Fig. 1).

When the direction of the transcriptional changes observed in the antennae was analyzed, we observed that the number of down-regulated genes was higher than that of up-regulated ones in all comparisons (Table 1). This was highest at older ages when $82 \%$ of DEGs decreased their expression, while it was lower than $66 \%$ during the first four days. All MA-plots reflected how down-regulation is predominant over upregulation in all comparisons (Additional files 4 and 5: Figs. S1 and S2).

A total of 86 sensory receptors, odorant carriers (OBPs and CSPs) and ODEs were identified as differentially expressed (Table 1 and Additional file 6: Table S4). Eighteen out of 116 OR genes had their expression significantly altered, representing $15 \%$ of the OR set. Interestingly, more than $30 \%$ of all transient receptor potential (TRP) channels presented significant changes in their expression profiles (5 out of 14 ) and a similar case was observed for pickpockets (PPKs) (3 out of 10). In contrast, only two IRs and two GRs were differentially expressed, i.e., less than $7 \%$ of each family showed modulated expression. The expression of most CSPs was affected by age, 15 out of 18 were found to be differentially expressed. For genes belonging to the OBP and ODE families, the percentage of DEGs was also high, with $13(46 \%)$ and 21 (65\%) members changing their expression during the first week after ecdysis.

The temporal pattern of gene expression observed was consistent with the overall changes described above, with the greatest alteration occurring between day 0 vs. day 2 (49 DEGs) followed by a decrease in the number of DEGs in subsequent comparisons (15, 7 and 3 DEGs, respectively). This situation was extremely clear with sensory receptors that varied almost exclusively between the first two days (16 DEGs). After day 2 only one odorant receptor (OR) and several sensory neuron membrane 
proteins (SNMPs) displayed changes in their expression levels (Table 2). In the case of odorant carriers and ODEs, the decrease observed in the number of DEGs progressed across all time points (Table 2). A higher number of targets were identified as DEGs in the grouped comparisons, suggesting that changes in some genes are cumulative and could not be detected by sequential comparisons. Down-regulation was always more frequent in all comparisons, except for the one between days 2 and 6 when upregulated genes were more abundant (Table 2 and Additional file 6: Table S4).

\section{Expression changes of odorant, ionotropic and gustatory receptors: examples of chemosensation regulated at the periphery}

A total of 18 ORs changed their antennal expression significantly during the first week after ecdysis (Additional file 6: Table S4). These receptors clustered in three profiles according to their expression (Fig. 1). The first (profile 1) was composed of four genes (RproOr17, RproOr39, RproOr96, and RproOr104), whose expression decreased with age. Fig. 3 depicts this clearly for RproOr17 and RproOr96, but also for RproOr39 and RproOr104, whose expression decreased significantly on day 2 . The second cluster defined a smaller group of genes that showed a significant down-regulation mostly after day 6 (profile 2). RproOr100 was the only OR in the second cluster (Fig. 2) and presented a significantly decreased expression on day 8 (Fig. 3). The last profile is composed of 13 ORs (Fig. 2), that increased their expression with bug age (profile 3). These genes presented two distinct patterns (Fig. 3): either a continuous rise in expression through the whole interval, e.g., RproOr30 and RproOr72, or an augmented expression at day 2 followed by stabilization, e.g., RproOr7 and RproOr18.

Transcriptional changes were very restricted for IRs and GRs, as only four of these genes changed their expression significantly (Fig. 3 and Additional file 6: Table S4). Two ionotropic receptors (IRs) belonging to profile 1, i.e. Rprolr93a and Rprolr107, decreased their expression drastically between days 0 and 2 (Fig. 3). A similar case was seen for the gustatory receptor (GR) RproGr14 that showed a significant decrease 
after day 4 (Fig. 3). Interestingly, the expression of RproGr26 rose steadily until day 6 (profile 3).

\section{Expression changes of PPK and TRP receptors}

The expression of only three PPKs receptors was affected by age (Fig. 2 and Additional file 6: Table S4). Rproppk23 and Rproppk28 belonging to profile 1 showed increased expression at the first age points (Fig. 4a). The expression pattern of the other PPK (Rprc014276) fit to profile 1, showing a reduction on day 2 (Fig. 4a).

Two different groups of TRP channels were identified by the clustering analysis according to their expression profiles (Fig. 2). RproNompC, RproPKD2, RproTrpA5b and RproTrpM belonged to profile 1, exhibiting a significant reduction on day 2 (Fig. $4 \mathrm{~b}$ and Additional file 6: Table S4). In contrast, Rprowtrw showed increased expression through the first half of the age interval, being clearly associated with profile 3 (Fig. 4b and Additional file 6: Table S4).

\section{Other membrane sensory proteins}

Three SNMPs were identified as DEGs distributed in all three clusters (Fig. 2 and Additional file 6: Table S4): RproSnmp1c (profile 1), which showed a decline until day 4; RproSnmp1d (profile 2), with a remarkable peak of expression at day 2 followed by a pronounced reduction through the following days; and RproSnmp2 (profile 3) with a significant increase after day 6 . Two additional genes clustered in profile 1: ammonium transporter 1 (RproAmt1) and the chemosensory protein B5 (RproCheB5), which presented a significantly decreased expression after day 0 (Fig. 4c).

\section{Expression changes of odorant carriers}

The effect of age on CSPs and OBPs was very clear, with $83 \%$ and $48 \%$ of them being differentially expressed, respectively (Additional file 6: Table S4). A first group including $10 \mathrm{CSPS}$ and 5 OBPs that fit into profile 1 had its expression reduced with age (Fig. 2). Dramatic examples of reduced expression could be seen with RproCsp6, 
RproCsp14, RproCsp17 or RproObp25 (Fig. 5). Three genes fit into profile 2, i.e., RproObp12, RproCsp10 and RproCsp13, showing a significant reduction only after day 6 (Fig. 5). Profile 3 was seen for 3 CSPs and 7 OBPs (Fig. 2) that displayed a rise in expression with age, as seen for RproCsp1, RproObp11, RproObp14 and RproObp19 (Fig. 5).

\section{Expression changes of odorant degrading enzymes}

A total of 4 secreted carboxylesterases esterases (Est) belonging to the pheromone/ hormone processing class and 17 members from cytochrome 4 (Сyp4) clade were classified as DEGs (Additional file 6: Table S4). Ten ODEs followed profile 1, showing very significant down-regulation with age, e.g., RproCyp4EM3, RproCyp4g, RproEst10 or RproCyp4h (Fig. 6). In contrast, 11 ODEs fit into profile 3, showing a dramatically increased expression with age, e.g., RproCyp4a, RproCyp4c, RproCyp4t, RproEst5 and RproCyp3097A9 (Fig. 6).

\section{Discussion}

Triatomine motivation for host searching is known to depend on age [30]. Nevertheless, it is not clear to which extent the central nervous system and peripheral sensory structures underlie the gradual increase seen through the first week after ecdysis. Our study shows that age induces dramatic changes in antennal gene expression that strongly correlate with the known age-dependent modulation of host search. Most of these transcriptional changes occur during the first two days after ecdysis, when more than 2,500 genes have their expression altered by more than $50 \%$. The set of sensory genes having significantly increased expression along age may include potential candidates underlying the increased host cue responsiveness shown by $R$. prolixus eight days after ecdysis. This is the first time such a maturation process undergone by the peripheral olfactory system is described in an hemimetabolous insect. 
The antennae of triatomines face major transcriptional expression changes during the first week, with a total of 6,120 genes $(54,7 \%$ of expressed genes) exhibiting significant modifications in their expression levels. The number of sensilla in triatomine antennae increases through development [31], supporting our results. In addition, part of these gene expression changes may relate to the known remodeling endured by sensilla during molting [32]. In all comparisons, a higher number of genes was significantly down-regulated, even though a relevant proportion of them underwent up-regulation (Table 1 ). This resembles the overall profiles described in the antennae of Ae. aegypti [8]. As shown by Fig. 2, there was no prevalence of downregulation in the case of differentially expressed sensory-related genes that present similar proportions of up/down-regulation. Indeed, the ratio between up and downregulated genes varied between age point comparisons, e.g. between days 2 and 4 the genes that increased their expression were more frequent (Table 1).

The first two days after ecdysis seem to present the most dramatic modulation of antennal gene expression in $R$. prolixus. This is true both in terms of number of genes affected and intensity of expression changes (see PCoA and MA plots). We propose that most of this process is related to the structural transformation that antennae undergo during moulting transforming them at molecular and physiological levels. However, part of this change seems to be related to the remodeling of sensory function (Table 2). Interestingly, behavioral changes related to host-searching, e.g. the capacity to respond to $\mathrm{CO} 2$ or heat, appear later between days 6 and 8 [30]. It is difficult to propose candidate genes underlying this increased capacity to respond to host cues based on our results, as most sensory-related genes that showed significant modulation seem to change in the first two days after ecdysis. However, a reduced subset of genes increase their antennal expression through the first week, being candidates for functional genetics studies (Additional file 6: Table S4). Whether the peripheral sensory system is functional in molecular terms at the end of the age interval studied, contributing to host cues responsiveness, or if these behavioral 
changes are triggered centrally cannot be determined based on our results. Indeed, antennae seem almost identical at the transcript level between day 6 and 8 (only 80 DEGs, Table 1 and Fig. 1). A similar pattern has been observed for the antennae of Ae. aegypti [8] and Anopheles coluzzi [9], where age-dependent molecular changes occur before the behavioral switch.

As expected according to their functional role, olfactory co-receptors displayed stable expression (Additional file 7: Table S5). Consistently, RproOrco was the most expressed gene among all ORs, but RproOr100 and RproOr20 seem to deserve attention because they were the ORs showing highest expression. This is consistent with previous antennal transcriptomes from fifth instar nymphs and adults of $R$. prolixus [29]. Regarding IR co-receptors, Rprolr25a was the most expressed followed by Rprolr8a and Rprolr76b (Additional file 7: Table S5), but transcripts of Rprolr93a and Rprolr75a were the most abundant also reinforcing the profiles reported by [29]. Interestingly, Ir93a has been described as a humidity sensor in D. melanogaster and a thermal receptor in An. gambiae [33], suggesting similar roles in triatomine host cue detection. The acetic acid responsiveness reported for Ir75a in D. melanogaster [34] suggests that detection of short-chain fatty acids may also be function of triatomine antennae. In fact, these compounds are known components of host odor, and in aggregation and alarm pheromones $[15,35,36]$.

Triatomines and mosquitoes are not attracted by hosts right after ecdysis, but show consistent responses to them a few days later [9, 30]. Indeed, mosquitoes also present antennal expression changes in sensory genes that correlate with this behavioral plasticity [8, 9, 27] In our study, two profiles split most genes that show significantly modulated expression across age points (Fig. 2), suggesting opposing temporal regulation profiles for their sensory functions. Profile 3 genes are candidates to underlie the modulation of responsiveness to host-associated cues because their expression is directly correlated to the increase in behavioral responsiveness seen during the first days after molting [30]. We suggest that OSNs expressing the 13 ORs 
included in profile 3 (Fig. 3 and Additional file 6: Table S4) should present increased responsiveness to their cognate ligands at the end of the first week after ecdysis. Unlike ORs, the expression of GRs and IRs was very stable after ecdysis. Only one GR (RproGr26) presented a significant increase in expression with age, peaking at day 6 (Fig. 3). This gene together with two other phylogenetically-related GRs (RproGr27 and RproGr28) are the most expressed genes of this family in bug antennae (Additional file 7: Table S5), suggesting that they possess fundamental roles for $R$. prolixus biology. We propose that the sensitivity of gustatory receptor neurons expressing RproGr26 should increase in correlation with its transcript abundance.

Regarding OBPs, CSPs and ODEs, a total of 20 genes increased their expression with age (Fig. 5 and 6). The first two families of soluble proteins have been proposed to mediate the delivery of odor molecules to receptors found in OSN membranes [37]. On the other hand, it has been shown that ODEs degrade odor molecules accumulating in the sensillar lymph, allowing the clearance of already detected molecules [38]. Considering these functional roles, we suggest that the detection of compounds targeted by this set of proteins would become more efficient through the first week after ecdysis.

Only two PPKs (RproPpk23 and RproPpk28) had their expression significantly increased as a function of post-ecdysis age (Fig. 4). In spite of the fact that a role in salt sensing has been described for these two PPKs in D. melanogaster [39] and Ae. aegypti [40], functional information is still scarce for triatomines. Nevertheless, it has been recently shown that RproPpk28 detects high salt concentration in the context of host skin recognition [41], suggesting that this sensory ability may show increased sensitivity at the end of the first week post-ecdysis.

The TRP channel water witch (wtrw) is an hygroreceptor that detects moist air in $D$. melanogaster [42]. Host skin and the breath of vertebrate hosts are sources of water vapour that is used by triatomines to oriente at short-range during host seeking [43, 44]. It is possible that this function was conserved across insect evolution and that 
triatomines also use it as a hygroreceptor mediating host recognition. We suggest that its up-regulation across age points in R. prolixus antennae (Fig. 4) would support this idea and indicates that detection of water vapor is not fully functional right after ecdysis.

Sensory genes whose expression significantly decreased with age (profile 1) were mainly CSPs (10) OBPs (5) and ODEs (11). Four ORs (RproOr17, RproOr39, RproOr96 and RproOr104), two IRs (Rprolr93a and Rprolr107), one GR (RproGr14), one PPK (Rprc014276) and four TRP channels (RproNompC, RproPKD2, RproTrpA5b and RproTrpM) presented this expression profile. Genes following this profile could mediate avoidance or behavioral inhibition to hosts as proposed for Or39, a receptor that detects human body odors that also presents a significant down-regulation between day 1 and 4 after molting in An. coluzzi [9]. An alternative hypothesis would be that the tasks related to these genes need to be fully functional right after ecdysis, having a relatively low turnover. Finally, it is known that the detection of alarm and aggregation signals is required at all ages and instars of triatomines. Genes that have high and stable expression in the antennae (Additional file 3: Table S3) might represent candidates for mediating similar functions.

\section{Conclusions}

The antennae of $R$. prolixus undergo a great molecular remodelling during the first days after ecdysis, a process that very likely transforms them at the physiological level. Part of this transformation is reflected in the transcript abundance of several sensory receptors and olfaction-related genes. We were able to identify a set of genes with sensory function whose expression increases across age, therefore becoming candidates to mediate the detection of host cues in this Chagas disease vector. However functional genetics studies, using RNA interference, CRISPR-cas9 technology or heterologous expression systems to allow their "deorphanization" are still necessary to transform them into feasible targets to manipulate triatomine behavior in the future. 


\section{Methods}

\section{Insects and RNA isolation}

Rhodnius prolixus were reared under controlled conditions $\left(26 \pm 1^{\circ} \mathrm{C}\right.$ temperature, $65 \pm 10 \%$ relative humidity and a $12 \mathrm{~h}: 12 \mathrm{~h}$ light/dark cycle) at the Instituto René Rachou - FIOCRUZ. Antennal samples were obtained from unfed fifth instar larvae along five different age points $(0,2,4,6$ and 8 days) after ecdysis. Each time point was replicated 7 times using 20 antennae per sample. Antennae were manually homogenized using sterilized pestles and total RNA was extracted using TRIzol ${ }^{\circledR}$ Reagent (Life Technologies, Carlsbad, CA, USA) according to the manufacturer's instructions. Then, extracted RNA was resuspended in $22 \mu \mathrm{L}$ of DEPC-treated water (Life Technologies), and its concentration determined at using Qubit (Thermo-Fisher Scientific). RNA integrity and quality were assessed by means of a $1 \%$ agarose electrophoresis gel and in Agilent 2100 Bioanalyzer (Agilent Technologies, Santa Clara, CA, USA).

\section{Illumina sequencing an quality control}

Library construction and sequencing services were performed by the Max Planck Genome Center (Cologne, Germany). RNA-Seq libraries were constructed using the TruSeq mRNA Sample Preparation Kit (Illumina, San Diego, CA). A total of 35 libraries were sequenced using Illumina HiSeq2500 from both ends of the molecules to a total read length of $150 \mathrm{bp}$ from each end. The raw sequence dataset is available with the NCBI-SRA Bioproject number PRJEB44111. The sequencing output has higher than 15 million reads in all libraries (Additional file 8: Table S6). The FASTQC software (bioinformatics.babraham.ac.uk/projects/fastqc/) was used to assess the read quality and the presence of Illumina sequencing adapters. Afterward, Illumina adapters and those bases from $5^{\prime}$ and $3^{\prime}$ ends with quality scores lower than 5 (TRAILING: 5 and LEADING: 5 parameters) were eliminated from the reads using Trimmomatic v0.32 in the paired-end mode [45]. The SLIDING-WINDOW parameter was fixed at 4:15 and only those reads longer than 50 base paired were kept for the next steps. 


\section{Mapping reads and differential gene expression analysis}

The trimmed reads (between 14 and 12 million reads per library) were mapped to the R. prolixus genome assembly (version RproC3.3 from VectorBase) by means of STAR v.2.6.0 [46] with default parameters and an edited General Feature Format file generated by [47]. The percentage of mapped reads was between 71 and $79 \%$ in all libraries, except for one replicate from day six with only $11 \%$ of mapped reads. Subsequently, the multicov command from the BEDTools suite v.2.27.0 was used to report the count of alignments per feature (Additional file 9: Table S7).

\section{Highly expressed genes along age}

The expression of each target gene was estimated as Transcripts Per Million (TPM) for each replicate and the geometric mean for each time point was calculated (Additional file 7: Table S5). Expression values of target genes were ranked for each time point and the top 50 genes were extracted. Finally, a consensus list of target genes present in the five rankings was elaborated.

\section{Differentially gene expression analysis}

Global analysis - EdgeR v3.30.1 package [48] was used to detect global changes across time in $R$. prolixus antennae. After the normalization (using calcNormFactors function) and estimation of gene-specific biological variation (using estimateDisp function) steps, normalized counts were analyzed using the GLM approach and the quasi-likelihood F-test. DEGs were identified considering a total of eight comparisons: sequential (day 0 vs. day 2 , day 2 vs. day 4, day 4 vs. day 6 and day 6 vs. day 8) and grouped (day 0 vs. all, days 0-2 vs. days 4-6-8, day 0-2-4 vs. day 6-8, all vs. day 8 ). Genes with low expression and/or high variation were filtered using the HTSFilter v.1.32 [49]. Those genes with an FDR $<0.05$ were considered as differentially expressed.

Pairwise comparisons - DESeq2 v.1.28.0 [50] was used to perform pairwise comparisons between the time points and groups mentioned above. First, the HTSFilter package was used to filter gene counts for each comparison. Following, each 
filtered dataset was used to identify DEGs using the apeg/m estimation from the IfcShrink function. This function is based on the "Approximate Posterior Estimation for Generalized Linear Model" (apeglm) method that uses an adaptive Bayesian shrinkage estimator to generate more accurate log2 fold-change values [51]. Finally, a DEG list was generated for each comparison based on $s$-values $<0.05$ to define the significance level and on a fold-change threshold $>1.5$. The output generated by the IfcShrink function was also used to elaborate MA-plots that show the mean of the normalized counts versus the $\log 2$ fold-changes for all genes tested.

Count data were transformed using the Variance Stabilizing Transformation (VST) for negative binomial data with a dispersion-mean trend [52], implemented in the vst function of DESeq2 (Additional file 10: Table S8). Following, transformed values of DEGs were extracted and used to generate heatmaps (using the pheatmap package in R) and box-plots. The transformed values of transcript expression were also used to calculate the euclidean distance between libraries and create a Principal Coordinate Analysis (PCoA) plot using the ade4 R package [10.18637/jss.v022.i04]. The impact of the variable day on the distances was measured by a PERMANOVA analysis using 999 permutations in R.

\section{List of abbreviations}

SNs: Sensory neurons

OSNs: Olfactory sensory neurons

GLM: General Linear Model

DEGs: Differentially expressed genes

OBPs: Odorant binding proteins

CSPs: Chemosensory proteins

ODEs: Odorant degrading enzymes

ORs: Odorant receptors

SNMPs: Sensory neuron membrane proteins 
IRs: Ionotropic receptors

GRs: Gustatory receptors

PPK: pickpockets

TRP: Transient receptor potential

Est: Secreted esterases

Cyp: Cytochromes

GRNs: Gustatory receptor neurons

TPM: Transcripts Per Million

VST: Variance Stabilizing Transformation

PcoA: Principal Coordinates Analysis

\section{Declarations}

\section{Ethics approval and consent to participate}

Not applicable

\section{Consent for publication}

Not applicable

\section{Availability of data and materials}

All data generated or analysed during this study are included in this published article and its supplementary information files.

\section{Competing interests}

The authors declare that they have no competing interests.

\section{Funding}

Authors are indebted to INCTEM (Project number: 465678/2014-9), , FIOCRUZ, CNPq (Project number: 308337/2015-8 and 311826/2019-9), and Agencia Nacional de Promoción Científica y Tecnológica (Project number: PICT 2016-3103). J.M.L.E. is a researcher from CONICET. E.G.W was further supported by the EXTEMIT-K project financed by Operational Programme Research, Development and Education at the Czech University of Life Sciences, Prague (CZ.02.1.01/0.0/0.0/15-003/0000433). 


\section{Authors' contributions}

M.G.L conceived the project. M.G.L, E.G.W, G.R.F, B.S.H. and J.M.L.E designed the experiments and performed data analyses. J.M.L.E. generated insects and RNA samples for RNA-Seq. J.M.L.E, E.G.W and G.R.F carried out the bioinformatic analyses and provided RNA-Seq data. All authors wrote the manuscript and provided comments on versions. All authors read and approved the final manuscript.

\section{Competing financial interests}

The authors declare that the research was conducted in the absence of any commercial or financial relationships that could be construed as a potential conflict of interest.

\section{Acknowledgements}

The authors acknowledge the Bioinformatics Platform of René Rachou Insitute for providing the computational resources to perform the analyses. 
Table 1. Number of differentially expressed genes identified in different time point comparisons performed along eight days after ecdysis. DEGs: Differentially expressed genes.

\begin{tabular}{lccccc}
\multicolumn{1}{c}{ Comparison } & Genes & DEGs & $\%$ & Up & Down \\
Day 0 vs. Day 2 & 10,681 & 1,738 & $16.27 \%$ & 652 & 1,086 \\
Day 2 vs. Day 4 & 10,803 & 527 & $4.88 \%$ & 177 & 350 \\
Day 4 vs. Day 6 & 14,358 & 188 & $1.31 \%$ & 28 & 160 \\
Day 6 vs. Day 8 & 10,630 & 80 & $0.75 \%$ & 14 & 66 \\
& & & & 1,11 & \\
Day 0 vs All & 11,252 & 2534 & $22.52 \%$ & 7 & 1,417 \\
Days 0 and 2 vs. All & 11,374 & 1815 & $15.96 \%$ & 685 & 1,130 \\
Days 0, 2 and 4 vs. Days 6 & & & & & \\
and 8 & 11,423 & 1171 & $10.25 \%$ & 350 & 821 \\
All vs. Day 8 & 11,252 & 620 & $5.51 \%$ & 167 & 453
\end{tabular}


Table 2. Number of differentially expressed genes from the sensory gene families identified in different temporal comparisons performed along eight days post-ecdysis. DEGs: differentially expressed genes; ORs: odorant receptors; GRs: gustatory receptors; IRs: ionotropic receptors; TRPs: transient receptor potential channels; SNMPs: sensory neuron membrane proteins; CHEB: Chemosensory proteins B; AmmT: Ammonium transporter; CSPs: Chemosensory proteins; OBPs: odorant binding proteins; ODEs: Odorant degrading enzymes. ODEs include cytochromes and esterases.

\begin{tabular}{|c|c|c|c|c|c|c|c|c|}
\hline Gene Family (DEGs) & $\begin{array}{c}\text { Day } 0 \\
\text { vs. } \\
\text { Day } 2\end{array}$ & $\begin{array}{c}\text { Day } 2 \\
\text { vs. } \\
\text { Day } 4\end{array}$ & $\begin{array}{c}\text { Day } 4 \\
\text { vs. } \\
\text { Day } 6\end{array}$ & $\begin{array}{c}\text { Day } 6 \\
\text { vs. } \\
\text { Day } 8\end{array}$ & $\begin{array}{c}\text { Day } 0 \\
\text { vs. } \\
\text { All }\end{array}$ & $\begin{array}{c}\text { Days 0-2 } \\
\text { vs. } \\
\text { All }\end{array}$ & $\begin{array}{c}\text { Days } 0,2,4 \\
\text { vs } \\
\text { Days } 6 \text { and } \\
8\end{array}$ & $\begin{array}{c}\text { All } \\
\text { vs. } \\
\text { Day } \\
8\end{array}$ \\
\hline ORs (18) & 7 & 0 & 0 & 1 & 16 & 4 & 2 & 2 \\
\hline GRs (2) & 0 & 0 & 0 & 0 & 1 & 0 & 1 & 0 \\
\hline IRs (2) & 2 & 0 & 0 & 0 & 2 & 2 & 0 & 0 \\
\hline TRPs (5) & 4 & 0 & 0 & 0 & 4 & 3 & 2 & 0 \\
\hline PPKs (3) & 2 & 0 & 0 & 0 & 1 & 1 & 0 & 0 \\
\hline SNMPS (3) & 1 & 1 & 1 & 0 & 1 & 1 & 1 & 2 \\
\hline CHEB (1) & 0 & 0 & 0 & 0 & 1 & 0 & 0 & 0 \\
\hline AmmT (1) & 0 & 0 & 0 & 0 & 1 & 0 & 0 & 0 \\
\hline CSPs (15) & 10 & 4 & 3 & 1 & 11 & 10 & 9 & 6 \\
\hline OBPs (13) & 9 & 2 & 1 & 0 & 9 & 7 & 6 & 5 \\
\hline ODEs (21) & 14 & 8 & 2 & 1 & 17 & 17 & 13 & 7 \\
\hline Total (84) & 49 & 15 & 7 & 3 & 64 & 45 & 34 & 22 \\
\hline Receptors (35) & 16 & 1 & 1 & 1 & 27 & 11 & 6 & 4 \\
\hline Carriers and ODEs (49) & 33 & 14 & 6 & 2 & 37 & 34 & 28 & 18 \\
\hline Up & 14 & 9 & 5 & 0 & 30 & 18 & 11 & 6 \\
\hline Down & 35 & 6 & 2 & 3 & 34 & 27 & 23 & 16 \\
\hline
\end{tabular}




\section{Figures and legends}

Fig. 1. Principal Coordinates Analysis Plot. The plot was created using the ade4 R package. The impact of the variable "age" on the distances was measured by a PERMANOVA analysis using 999 permutations in R.

Fig. 2. Effect of age on the antennal transcription of sensory-related genes. The heatmap was created using transformed count data (generated with vst function in DESeq2 and included in Additional file 10: Table S8) as input of the pheatmap R package. This calculated a z-score for each gene and plotted it by means of a color scale, where blue/red represent lowest/highest expression. Dendrogram was created with hierarchical clustering among genes based on their Euclidean distances and the Ward method for clustering.

Fig. 3. Box-plots of differentially expressed odorant, ionotropic and gustatory receptors along five age points post-ecdysis. Gene expression is represented as Variance Stabilizing Transformed (VST) counts that were obtained from count data and the vst function from DESeq2. VST counts are reported in Additional file 10: Table S8.

Fig 4. Box-plots of differentially expressed pickpocket, transient potential receptor channels and other sensory receptors along five age points postecdysis. Gene expression is represented as Variance Stabilizing Transformed (VST) counts that were obtained from count data and the vst function from DESeq2. VST counts are reported in Additional file 10: Table S8.

Fig. 5. Box-plots of differentially expressed odorant binding and chemosensory proteins along five age points post-ecdysis. Gene expression is represented as Variance Stabilizing Transformed (VST) counts that were obtained from 
count data and the vst function from DESeq2. VST counts are reported in Additional file 10: Table S8.

Fig. 6. Box-plots of differentially expressed odorant degrading enzymes along five age points post-ecdysis. Gene expression is represented as Variance Stabilizing Transformed (VST) counts that were obtained from count data and the vst function from DESeq2. VST counts are reported in Additional file 10: Table S8.

\section{Additional files}

Additional file 1: Table S1. Results of PERMANOVA Pairwise comparisons. Df: Degrees of freedom;

Additional file 2: Table S2. Results of the differential gene expression analysis using the GLM approach performed in EdgeR. FC: Fold change; CPM: Count per million; FDR: False Discovery Rate.

Additional file 3: Table S3. Sensory genes showing highest expression along 8 days post-ecdysis. This list was generated from the consensus of the 50 most expressed sensory genes in each time point as described in Supplementary Table S5. Gene expression (expressed as TPM) was calculated using the geometric mean of the five time points (obtained with the geometric mean of each technical replicate per time point). TPM: Transcripts Per Million; DEG: Differentially Expressed Gene.

Additional file 4: Fig. S1. MA-plots of Day 0 vs. Day 2 (top left), Day 2 vs Day 4 (top right), Day 4 vs Day 6 (bottom left) and Day 6 vs Day 8 (bottom right) representing log fold-change versus mean expression of normalized counts. Differentially expressed genes are shown in red. The blue line represents a logFold change cut-off $(0.583$, equivalent to a $50 \%$ fold-change) used to identify differentially expressed genes (DEGs). 
Additional file 5: Fig. S2. MA-plots of Day 0 vs. All (top left), Days 0 and 2 vs All (top right), Days 0,4,6 vs Days 6 and 8 (bottom left) and Day All vs Day 8 (bottom right) representing log fold-change versus mean expression of normalized counts. Differentially expressed genes are shown in red. The blue line represents a logFold change cut-off $(0.583$, equivalent to a $50 \%$ fold-change) used to identify differentially expressed genes (DEGs).

Additional file 6: Table S4. Results of the differential gene expression analysis performed in DESeq2.

Additional file 7: Table S5. Transcripts Per Million values for sensory genes in the 34 libraries and geometric mean for each time point (green) and the five time points.

Additional file 8: Table S6. Raw, trimmed and mapped reads, including percentages, for each library.

Additional file 9: Table S7. Raw counts for each gene in the 35 libraries.

Additional file 10: Table S8. Variance Stabilizing Transformed counts for each gene in the 34 libraries. 
1. Horn E, Knapp A. On the invariance of visual stimulus efficacy with respect to variable spatial positions. J Comp Physiol A 1984 1544. 1984;154:555-67. doi:10.1007/BF00610169.

2. Lazzari CR. Chapter 1 Orientation Towards Hosts in Haematophagous Insects: An Integrative Perspective. Adv In Insect Phys. 2009;37:1-58.

3. Guerenstein PG, Lazzari CR. Host-seeking: How triatomines acquire and make use of information to find blood. Acta Trop. 2009;110:148-58.

4. Van der Goes van Naters WM, Den Otter CJ, Maes FW. Olfactory Sensitivity in Tsetse Flies: a Daily Rhythm. Chem Senses. 1998;23:351-7. doi:10.1093/CHEMSE/23.3.351.

5. Tanoue S, Krishnan P, Krishnan B, Dryer SE, Hardin PE. Circadian clocks in antennal neurons are necessary and sufficient for olfaction rhythms in Drosophila. Curr Biol. 2004;14:638-49.

6. Krishnan P, Chatterjee A, Tanoue S, Hardin PE. Spike Amplitude of Single-Unit Responses in Antennal Sensillae Is Controlled by the Drosophila Circadian Clock. Curr Biol. 2008;18:803-7.

7. Page TL, Koelling E. Circadian rhythm in olfactory response in the antennae controlled by the optic lobe in the cockroach. J Insect Physiol. 2003;49:697-707.

8. Tallon AK, Hill SR, Ignell R. Sex and age modulate antennal chemosensory-related genes linked to the onset of host seeking in the yellow-fever mosquito, Aedes aegypti. Sci Rep. 2019;9:1-13.

9. Omondi AB, Ghaninia M, Dawit M, Svensson T, Ignell R. Age-dependent regulation of host seeking in Anopheles coluzzii. Sci Rep. 2019;9.

10. Klowden MJ, Lea AO. Abdominal distention terminates subsequent host-seeking behaviour of Aedes aegypti following a blood meal. J Insect Physiol. 1979;25:583-5.

11. Bodin A, Vinauger C, Lazzari CR. Behavioural and physiological state dependency of host seeking in the blood- sucking insect Rhodnius prolixus. 2009;:2386-93.

12. Klowden MJ, Lea AO. Blood Meal Size as a Factor Affecting Continued Host-Seeking by Aedes Aegypti (L.). Am J Trop Med Hyg. 1978;27:827-31. doi:10.4269/AJTMH.1978.27.827.

13. Klowden MJ, Davis EE, Bowen MF. Role of the fat body in the regulation of host-seeking behaviour in the mosquito, Aedes aegypti. J Insect Physiol. 1987;33:643-6.

14. Lorenzo MG, Lazzari CR. The spatial pattern of defaecation in Triatoma infestans and the role of faeces as a chemical mark of the refuge. J Insect Physiol. 1996;42:903-7.

15. Mota T, Vitta ACR, Lorenzo-Figueiras AN, Barezani CP, Zani CL, Lazzari CR, et al. A Multi-species Bait for Chagas Disease Vectors. PLoS Negl Trop Dis. 2014;8:e2677. doi:10.1371/JOURNAL.PNTD.0002677.

16. Cruz-Lopez L, Malo EA, Rojas JC. Aggregation pheromone in five species of Triatominae (Hemiptera: Reduviidae). Mem Inst Oswaldo Cruz. 1993;88:535-9. doi:10.1590/S0074-02761993000400006.

17. Figueiras ANL, Lazzari CR. Temporal change of the aggregation response in Triatoma infestans. Mem Inst Oswaldo Cruz. 2000;95:889-92. doi:10.1590/S0074-02762000000600026.

18. Palottini F, Manrique G. Compounds released by disturbed adults of the haematophagous bug Triatoma infestans (Hemiptera: Reduviidae): behavioural effects of single compounds and binary mixtures. Physiol Entomol. 2016;41:234-40. 
19. Gadenne C, Barrozo RB, Anton S. Plasticity in Insect Olfaction: To Smell or Not to Smell? Annu Rev Entomol. 2016;61:317-33.

20. Reisenman CE. Hunger is the best spice: Effects of starvation in the antennal responses of the bloodsucking bug Rhodnius prolixus. J Insect Physiol. 2014;71 September:8-13. doi:10.1016/j.jinsphys.2014.09.009.

21. Davis EE. Regulation of sensitivity in the peripheral chemoreceptor systems for host-seeking behaviour by a haemolymph-borne factor in Aedes aegypti. J Insect Physiol. 1984;30:179-83.

22. Bohbot JD, Durand NF, Vinyard BT, Dickens JC. Functional development of the octenol response in Aedes aegypti. Front Physiol. 2013;4 MAR March:1-8.

23. Hill SR, Taparia T, Ignell R. Regulation of the antennal transcriptome of the dengue vector, Aedes aegypti, during the first gonotrophic cycle. BMC Genomics. 2021;22:1-19.

24. Martel V, Anderson P, Hansson BS, Schlyter F. Peripheral modulation of olfaction by physiological state in the Egyptian leaf worm Spodoptera littoralis (Lepidoptera: Noctuidae). J Insect Physiol. 2009;55:793-7.

25. Saveer AM, Kromann SH, Birgersson G, Bengtsson M, Lindblom T, Balkenius A, et al. Floral to green: Mating switches moth olfactory coding and preference. Proc R Soc B Biol Sci. 2012;279:2314-22.

26. Tallon AK, Lorenzo MG, Moreira LA, Villegas LEM, Hill SR, Ignell R. Dengue infection modulates locomotion and host seeking in Aedes aegypti. PLoS Negl Trop Dis. 2020;14:e0008531. doi:10.1371/JOURNAL.PNTD.0008531.

27. Hill SR, Taparia T, Ignell R. Regulation of the antennal transcriptome of the dengue vector, Aedes aegypti, during the first gonotrophic cycle. BMC Genomics 2021 221. 2021;22:1-19. doi:10.1186/S12864020-07336-W.

28. Rinker DC, Pitts RJ, Zhou X, Suh E, Rokas A, Zwiebel LJ. Blood meal-induced changes to antennal transcriptome profiles reveal shifts in odor sensitivities in Anopheles gambiae. Proc Natl Acad Sci U S A. 2013;110:8260-5.

29. Latorre-Estivalis JM, Robertson HM, Walden KKO, Ruiz J, Gonçalves LO, Guarneri AA, et al. The molecular sensory machinery of a Chagas disease vector: Expression changes through imaginal moult and sexually dimorphic features. Sci Rep. 2017.

30. Bodin A, Vinauger C, Lazzari CR. State-dependency of host-seeking in Rhodnius prolixus: The postecdysis time. J Insect Physiol. 2009;55:574-9.

31. Gracco M, Catalá S. Inter-specific and Developmental Differences on the Array of Antennal Chemoreceptors in Four Species of Triatominae (Hemiptera: Reduviidae). Mem Inst Oswaldo Cruz. 2000;95:67-74.

32. GNATZY W, TAUTZ J. Sensitivity of an insect mechanoreceptor during moulting. Physiol Entomol. 1977;2:279-88. doi:10.1111/J.1365-3032.1977.TB00118.X.

33. Greppi C, Laursen WJ, Budelli G, Chang EC, Daniels AM, Giesen L van, et al. Mosquito heat seeking is driven by an ancestral cooling receptor. Science (80- ). 2020;367:681-4. doi:10.1126/SCIENCE.AAY9847.

34. Prieto-Godino LL, Rytz R, Bargeton B, Abuin L, Arguello JR, Peraro MD, et al. Olfactory receptor pseudo-pseudogenes. Nature. 2016;539:93-7. doi:10.1038/nature19824.

35. Barrozo RB, Minoli SA, Lazzari CR. Circadian rhythm of behavioural responsiveness to carbon dioxide in the blood-sucking bug Triatoma infestans (Heteroptera: Reduviidae). J Insect Physiol. 2004;50:249-54. 
36. Manrique G, Vitta ACR, Ferreira RA, Zani CL, Unelius CR, Lazzari CR, et al. Chemical Communication in Chagas Disease Vectors. Source, Identity, and Potential Function of Volatiles Released by the Metasternal and Brindley’s Glands of Triatoma infestans Adults. J Chem Ecol 2006329. 2006;32:2035-52. doi:10.1007/S10886-006-9127-7.

37. Leal WS. Odorant reception in insects: Roles of receptors, binding proteins, and degrading enzymes. Annu Rev Entomol. 2013;58 September 2012:373-91.

38. Steiner C, Chertemps T, Maïbèche M. Diversity of Biotransformation Enzymes in Insect Antennae: Possible Roles in Odorant Inactivation and Xenobiotic Processing. Olfactory Concepts Insect Control Altern to Insectic. 2019;:115-45. doi:10.1007/978-3-030-05165-5_5.

39. Jaeger AH, Stanley M, Weiss ZF, Musso PY, Chan RCW, Zhang H, et al. A complex peripheral code for salt taste in drosophila. Elife. 2018;7:1-30.

40. Matthews BJ, Younger MA, Vosshall LB. The ion channel ppk301 controls freshwater egg-laying in the mosquito aedes aegypti. Elife. 2019;8:1-27.

41. Pontes G, Latorre-Estivalis J, Gutiérrez M, Cano A, Astrada MB de, Lorenzo M, et al. Salty surfaces deter feeding in a blood-sucking disease vector. bioRxiv. 2021;:2021.03.22.436426. doi:10.1101/2021.03.22.436426.

42. Liu L, Li Y, Wang R, Yin C, Dong Q, Hing H, et al. Drosophila hygrosensation requires the TRP channels water witch and nanchung. Nature. 2007;450:294-8.

43. Wigglesworth BYVB, Gillett JD. The Function of the Antennae in Rhodnius prolixus (Hemiptera) and the Mechanism of Orientation to the Host. J Exp Biol. 1934;11:120-39.

44. Barrozo RB, Manrique G, Lazzari CR. The role of water vapour in the orientation behaviour of the blood-sucking bug Triatoma infestans (Hemiptera, Reduviidae). J Insect Physiol. 2003;49:315-21.

45. Bolger AM, Lohse M, Usadel B. Trimmomatic: a flexible trimmer for Illumina sequence data. Bioinformatics. 2014;30:2114-20. doi:10.1093/BIOINFORMATICS/BTU170.

46. Honarbakhsh S, Schilling RJ, Finlay M, Keating E, Ullah W, Hunter RJ. STAR mapping method to identify driving sites in persistent atrial fibrillation: Application through sequential mapping. J Cardiovasc Electrophysiol. 2019;30:2694-703. doi:10.1111/JCE.14201.

47. Latorre-Estivalis JM, Sterkel M, Ons S, Lorenzo MG. Transcriptomics supports local sensory regulation in the antenna of the kissing-bug Rhodnius prolixus. BMC Genomics. 2020.

48. Chen Y, Mccarthy D, Robinson M, Smyth GK. edgeR : differential expression analysis of digital gene expression data User's Guide. 2014; March.

49. Rau A, Gallopin M, Celeux G, Jaffrézic F. Data-based filtering for replicated high-throughput $\begin{array}{llll}\text { transcriptome } \quad \text { sequencing } & \text { 2013;29:2146-52. }\end{array}$ doi:10.1093/BIOINFORMATICS/BTT350.

50. Love MI, Anders S, Huber W. Differential analysis of count data - the DESeq2 package. 2014. http://biorxiv.org/lookup/doi/10.1101/002832\%5Cnhttp://dx.doi.org/10.1186/s13059-014-0550-8.

51. Zhu A, Ibrahim JG, Love MI. Heavy-tailed prior distributions for sequence count data: removing the noise and preserving large differences. Bioinformatics. 2019;35:2084-92. doi:10.1093/BIOINFORMATICS/BTY895. 
bioRxiv preprint doi: https://doi.org/10.1101/2021.09.02.458747; this version posted September 2, 2021. The copyright holder for this preprint (which was not certified by peer review) is the author/funder, who has granted bioRxiv a license to display the preprint in perpetuity. It is made available under aCC-BY-NC-ND 4.0 International license.

52. Anders S, Huber W. Differential expression analysis for sequence count data. Nat Preced 2010. 2010;:1-

1. doi:10.1038/npre.2010.4282.1. 
CSP1

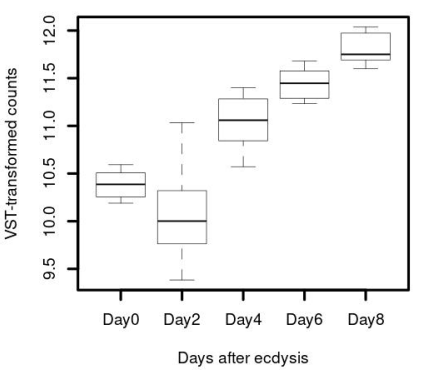

CSP5

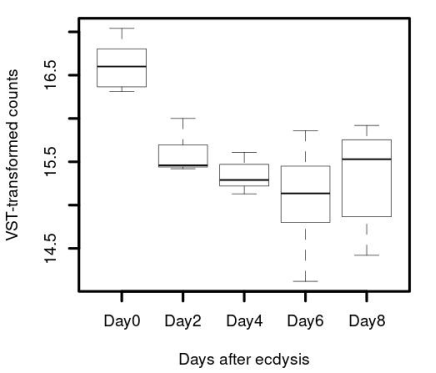

Days after ecdysis

CSP14

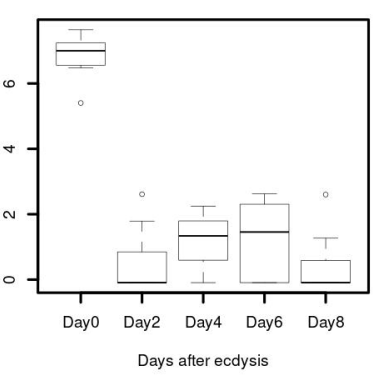

OBP11

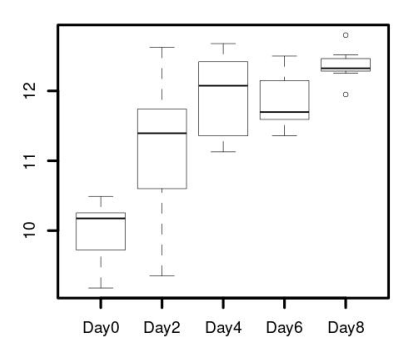

Days after ecdysis

OBP4

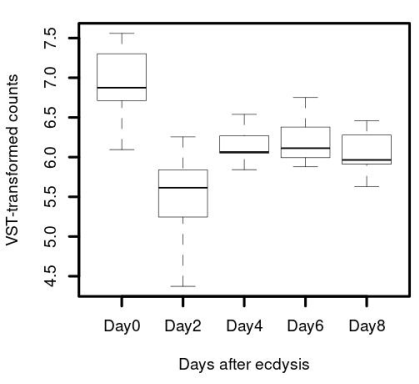

OBP10

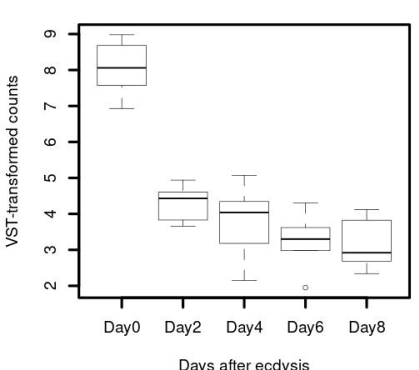

$\operatorname{csp} 3$

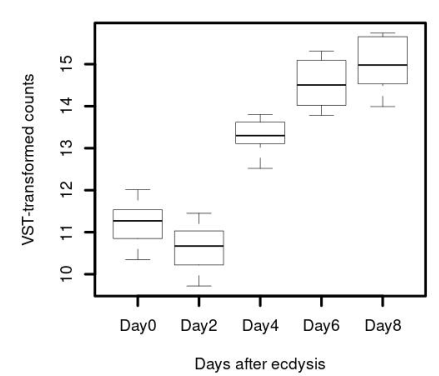

CSP6

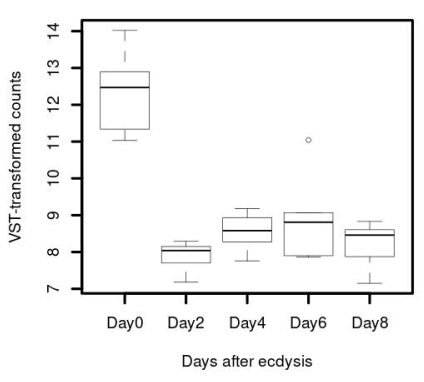

CSP15

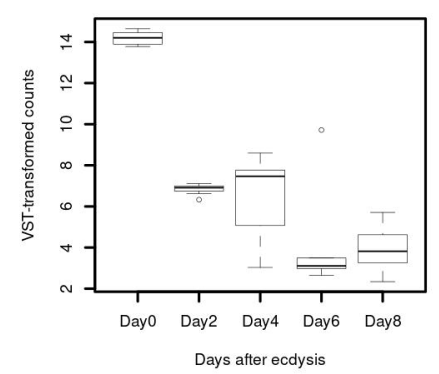

OBP14

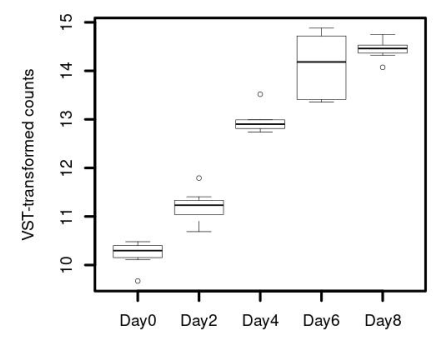

Days after ecdysis

OBP13

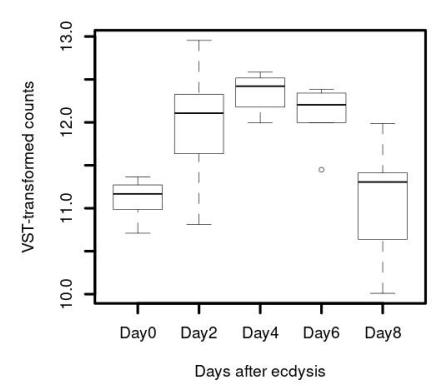

овP12

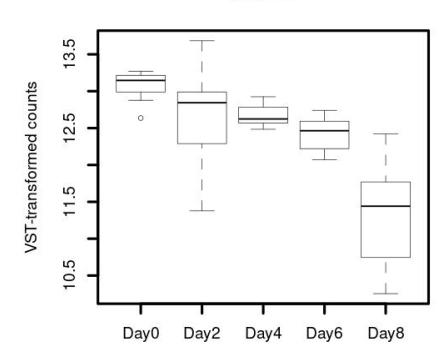

Days after ecdysis

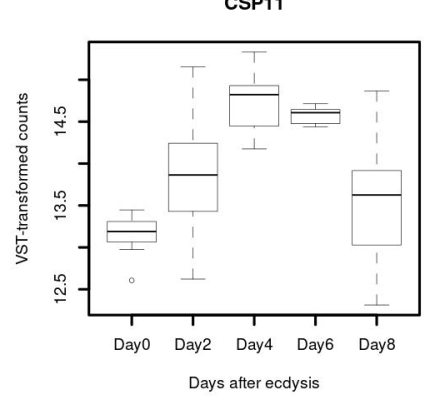

CSP7

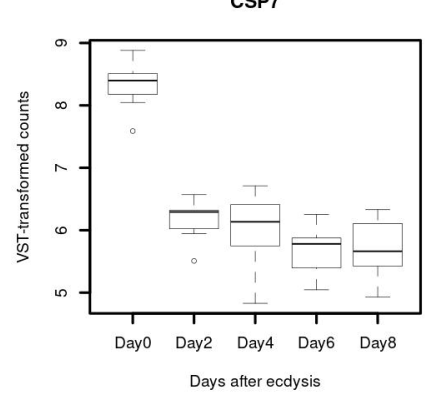

CSP16

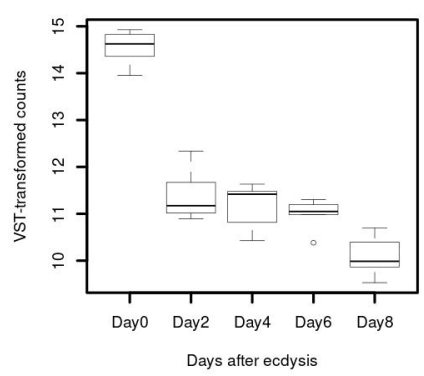

OBP19

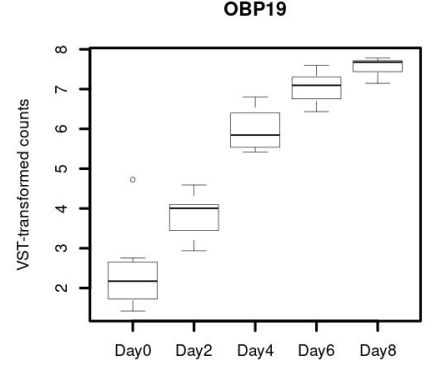

Days after ecdysis

OBP18
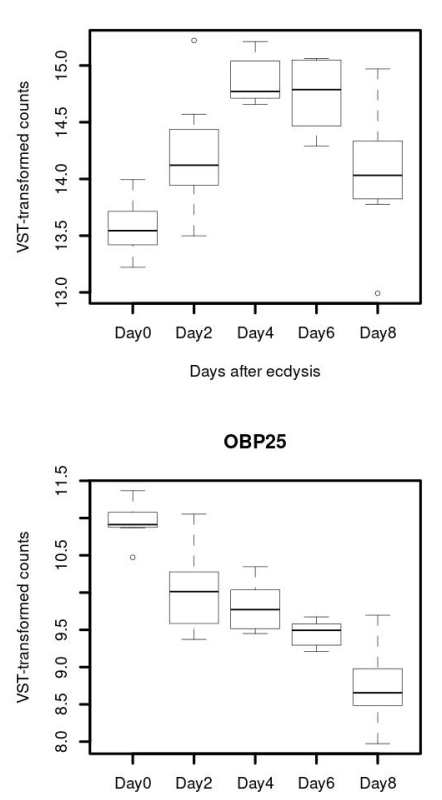

Days after ecdysis

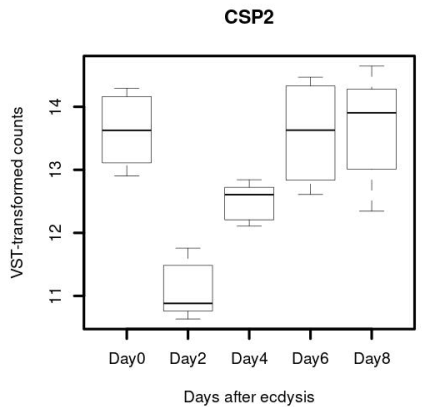

CSP4
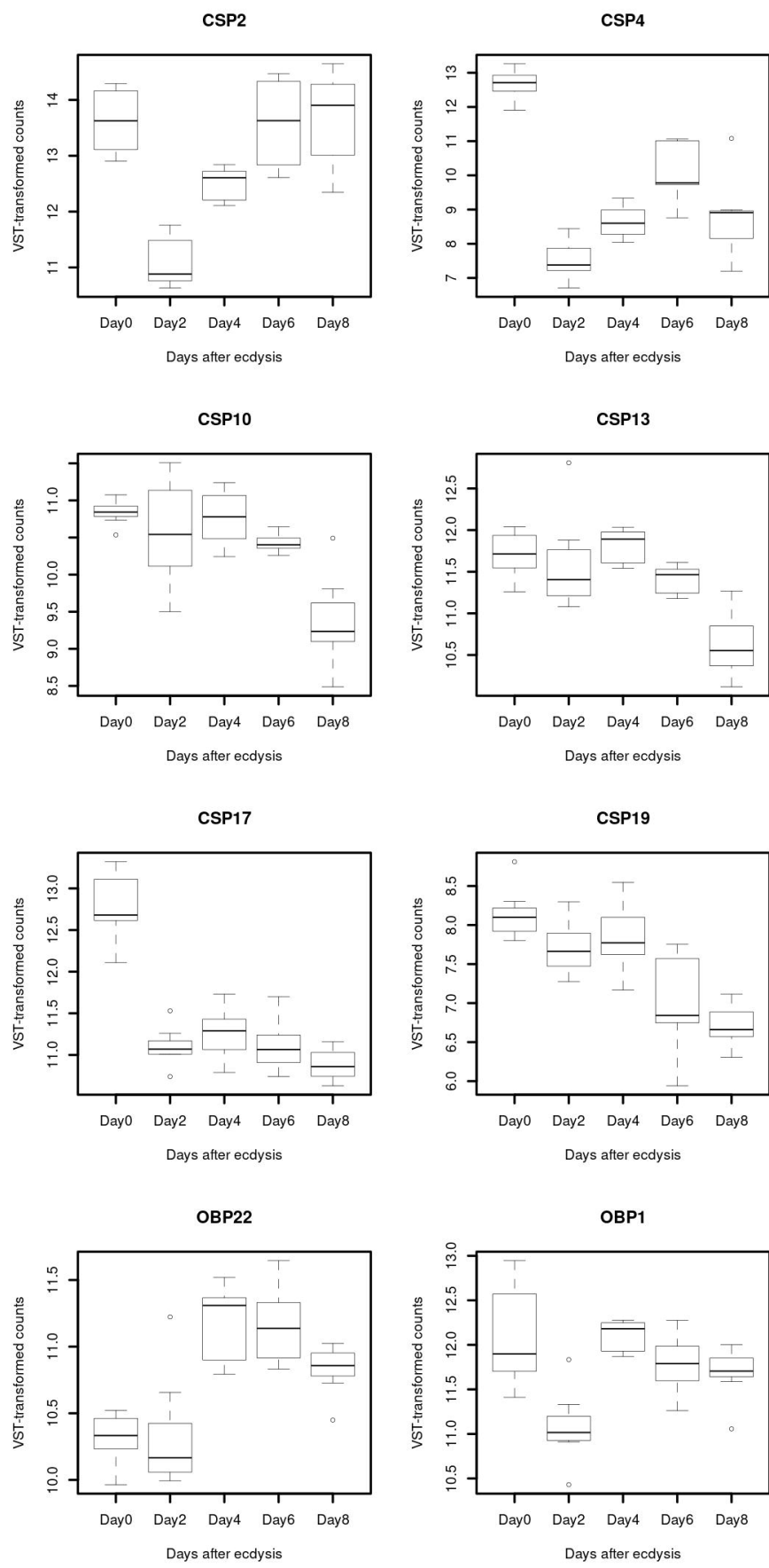

OBP21

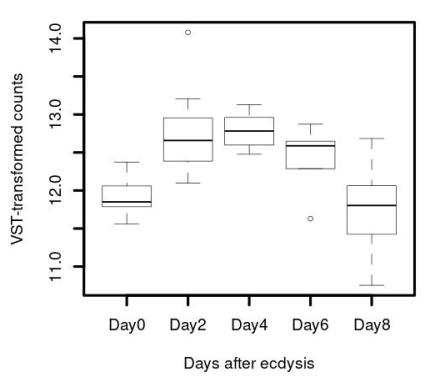

OBP3

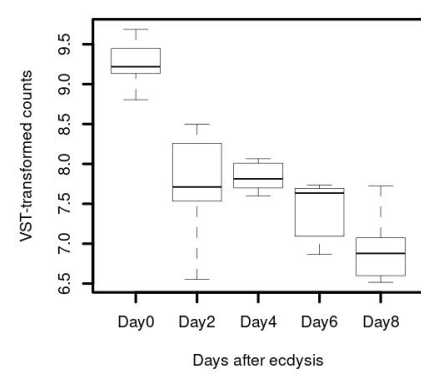


SNMP1C

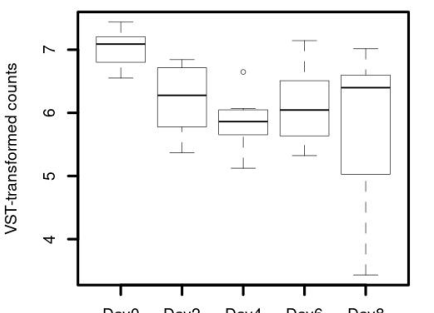

Day0 Day2 Day4 Day6 Day8

Days after ecdysis

RPRC014276

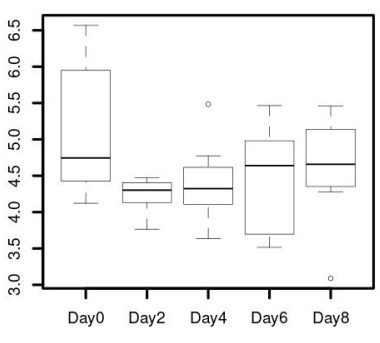

Days after ecdysis

WTRW

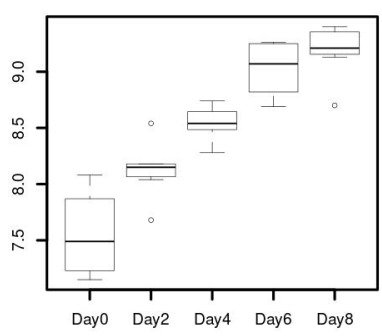

Days after ecdysis
SNMP1d

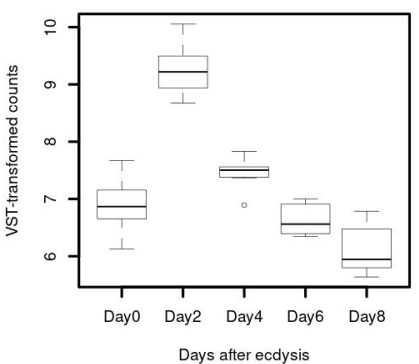

NOMPC

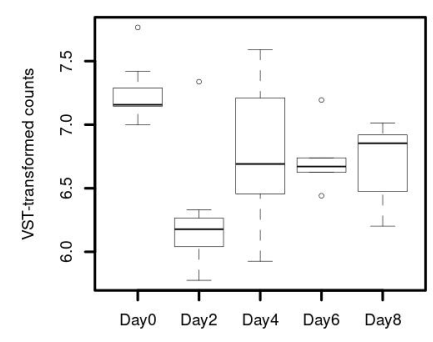

Days after ecdysis

AMT1

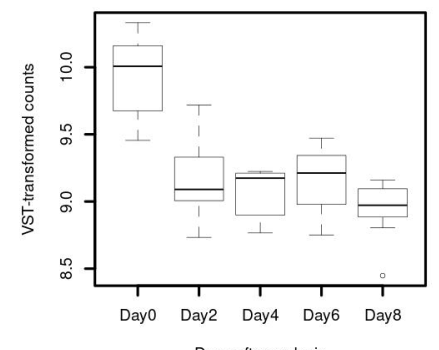

Days after ecdysis
SNMP2

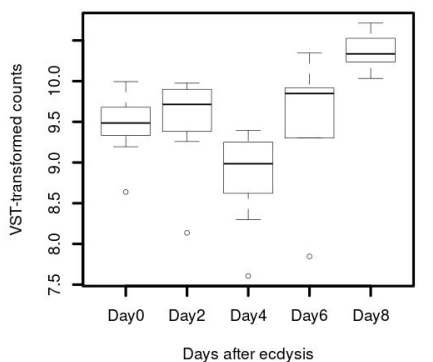

PKD2

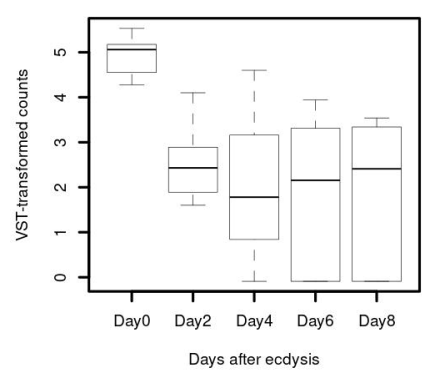

CHEB5

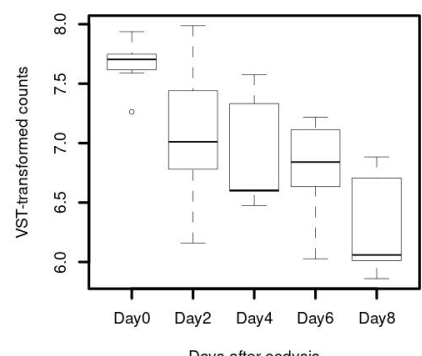

PPK23

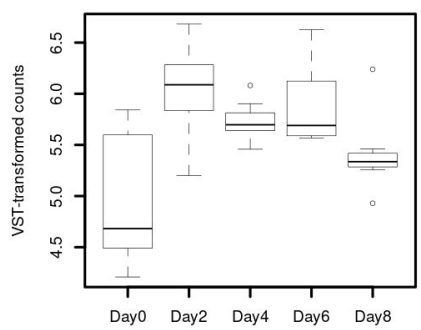

Days after ecdysis

TRPA5b

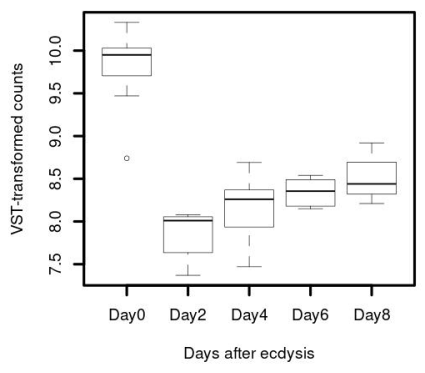

PPK28

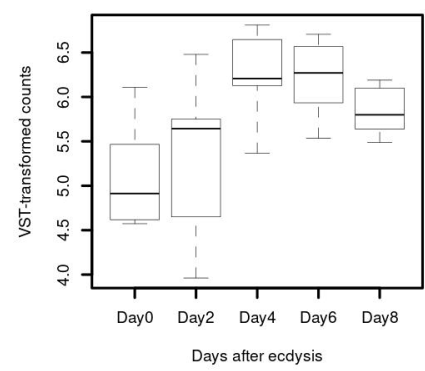

TRPM

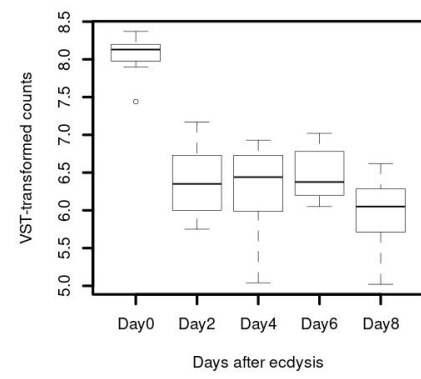




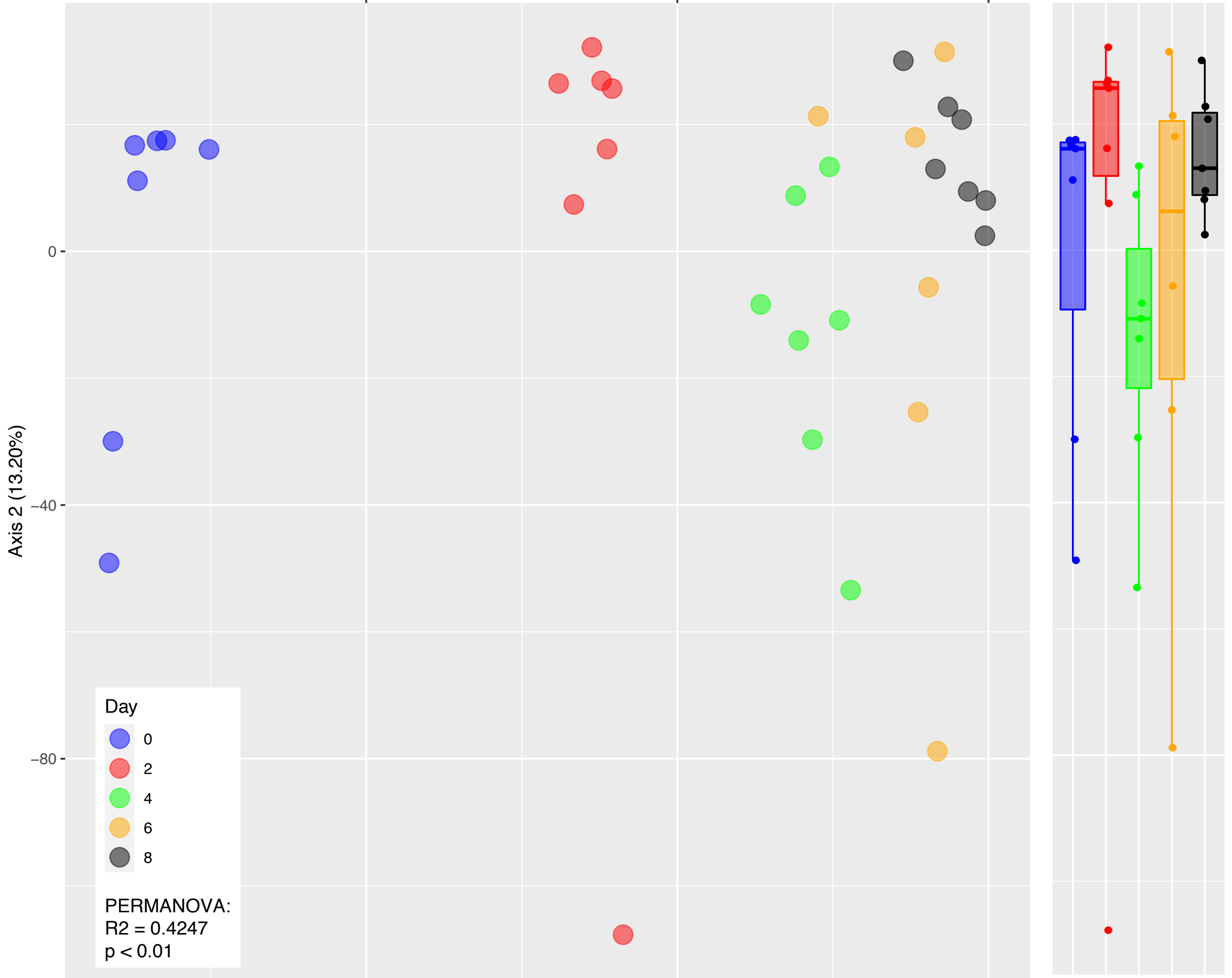

Day

타 0

타 2

타 4 